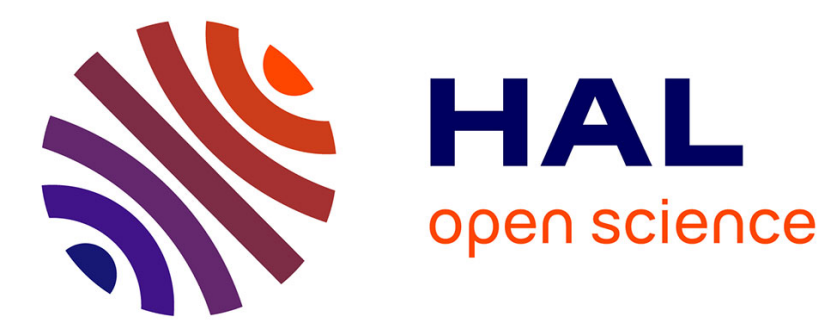

\title{
Hemisphere m-line spectroscopy and its application to birefringent KTiOPO4 planar waveguides.
}

\author{
Jochen Fick, Bertrand Ménaert, Julien Zaccaro, Paul Moretti
}

\section{To cite this version:}

Jochen Fick, Bertrand Ménaert, Julien Zaccaro, Paul Moretti. Hemisphere m-line spectroscopy and its application to birefringent KTiOPO4 planar waveguides.. Optics Communications, 2007, 270, pp.229. 10.1016/j.optcom.2006.09.017 . hal-00154199

\section{HAL Id: hal-00154199 \\ https://hal.science/hal-00154199}

Submitted on 13 Jun 2007

HAL is a multi-disciplinary open access archive for the deposit and dissemination of scientific research documents, whether they are published or not. The documents may come from teaching and research institutions in France or abroad, or from public or private research centers.
L'archive ouverte pluridisciplinaire HAL, est destinée au dépôt et à la diffusion de documents scientifiques de niveau recherche, publiés ou non, émanant des établissements d'enseignement et de recherche français ou étrangers, des laboratoires publics ou privés. 


\title{
Hemisphere $m$-line spectroscopy and its application to birefringent $\mathrm{KTiOPO}_{4}$ planar waveguides.
}

\author{
Jochen Fick \\ Laboratoire de Spectrométrie Physique, UMR 5588 CNRS - Univesité Joseph Fourier Grenoble I, BP 87, \\ 38402 St. Martin d'Hères, France \\ Bertrand Ménaert, Julien Zaccaro \\ Laboratoire de Cristallographie, UPR 5031 CNRS, BP 166, 38042 Grenoble, France
}

Paul Moretti

Laboratoire de Physico-Chimie des Matériaux Luminescentes, UMR 5620 CNRS - Université Claude Bernard

Lyon I, 69622 Villeurbanne, France

\begin{abstract}
A modified $m$-line technique, based on the use of a high index hemisphere instead of a coupling prism is presented. It allows fast characterization of the optical properties as a function of the propagation direction inside a planar waveguide. Results of an ion-implanted KTP waveguide are presented and compared to prism measurements. It is shown that this techniques enhances the precision of the waveguide parameters determination and allows to study advanced features such as strong mode hybridization.
\end{abstract}

PACS codes: 42.70. Mp: Nonlinear optical crystals

42.82. Et: Waveguides - couplers

42.87. -d: Optical testing techniques

48.25. Lc: Birefringence

\section{Introduction}

The classic $m$-line spectroscopy, based on a coupling prism, is a widely used technique for the characterization of planar optical waveguides, allowing to test for waveguiding, determine the main waveguide parameters and to get an estimation of the waveguide losses. It allows fast characterization without any sample preparation, such as end-face polishing in the case of butt coupling. The measurement is made on a small waveguide region, allowing to test for homogeneity and/ or to work with mediocre quality samples during waveguide fabrication optimization. The $m$ line measurement does not cause any damage, allowing further sample processing. Automatized $m$ line systems are commercially available for some years.

The $m$-line spectroscopy was developed in the seventies by P.K. Tien and R. Ulrich. They complete the experimental observation of waveguide modes in semiconductor thin films [1] by the theoretical treatment [2] of a prism coupler using a plain wave approach. Later, this theory was extended to non linear distributed couplers $[3,4]$ allowing to simulate dynamical and non linear effects in prism 
and grating couplers [5].

The $m$-lines are mostly used for the isotropic linear waveguides. However, a non linear versions of the $m$-lines was developed to study the optical Kerr effect of thin film waveguides [6-8]. Further on, $m$-lines were used for the characterization of anisotropic waveguides by measuring the waveguide modes as a function of the propagation direction in the waveguide plane. This method was used for the investigation of induced anisotropy of proton-exchanged lithium niobate waveguides [9], KTP ion-implanted waveguides [10], liquid crystal films [11] or the anisotropy of sputtered titanium or hafnium oxide films [12,13]. The variation of the propagation direction was obtained by demounting the sample and remounting it slightly tilted. This is a awkward and time consuming procedure. Moreover, it is difficult to reproduce exactly the same coupling conditions at the same spot of the planar waveguide and the determination of the exact propagation direction is difficult. These problems can be overcome by replacing the coupling prism by a hemisphere and adding a rotation stage to allow rotation of the sample around the waveguide normal. In this paper, the hemisphere $m$-line set-up will be presented. It will be used for the investigation of the TE-TM mode hybridization in an ion-implanted KTP waveguide.

\section{Experimental set-up}

The hemispherical $m$-line set-up is depicted in Fig. 1. A laser diode emitting at $980 \mathrm{~nm}$ is used as light source followed by a ball lens $(f=4 \mathrm{~mm})$ for beam collimation. A Glan prism polarizer and a $\lambda / 2$ plate allows to control the beam polarization by turning the $\lambda / 2$ plate. No shift of the incident beam was observed when changing from TE to TM polarization. The main complication when using the hemisphere instead of the coupling prism is the fact that the hemisphere acts as a lens with a short focal length $\left(f_{\mathrm{hs}} \cong 4 \mathrm{~mm}\right)$. To obtain a well collimated laser beam at the base of the hemisphere, the beam has to be focused near the hemisphere surface. Gaussian beam theory was used to simulate the beam propagation. A telescope with $f_{l}=80 \mathrm{~mm}$ and $f_{2}=100 \mathrm{~mm}$ leads to a beam waist of $\omega=0.2 \mathrm{~mm}$ and a Rayleigh length of $\mathrm{z}_{\mathrm{r}}=130 \mathrm{~mm}$. This high $\mathrm{z}_{\mathrm{r}}$ value assures a well collimated incident beam and allows noncritical alignment.

The sample is mounted in the center of a theta/2theta two circle goniometer actuated by two step motors. The reflected intensity is recorded using a silicon photo-diode. The aperture of the detector is enlarged by a lens to enhance the capture of the reflected beam. The waveguide is pressed onto the hemisphere by means of a micrometer screw. A index matching liquid with $n_{\lambda=589 \mathrm{~nm}}=1.58$ is used to lower the effective thickness of the gap between the hemisphere and the sample. A manual rotation stage allows the rotation around the waveguide normal.

The hemisphere is obtained by polishing a commercial $8 \mathrm{~mm}$ ball lens made of S-LAH79 high index glass from Ohara. The glass refractive index is $n_{\mathrm{hs}}=1.9703$ at $980 \mathrm{~nm}$, which is well adapted for KTP waveguides. A coupling prism of the same glass was used to test the performance of the hemisphere coupler.

\section{KTP waveguide fabrication}

A planar waveguide was fabricated by $\mathrm{He}^{+}$ion implantation of a $x$-cut KTP substrate from Cristal Laser. Two subsequent implantations were applied at energies of 1.5 and $1.45 \mathrm{MeV}$ with a fluence 
of $0.75 \cdot 10^{16}$ ions $/ \mathrm{cm}^{2}$ each. The estimated waveguide depth was $4 \mu \mathrm{m}$. A post irradiation annealing at $250^{\circ} \mathrm{C}$ for one hour was applied to cure eventual electronic defects in the waveguide. Details on the waveguide fabrication process can be found in Ref. [10].

The propagation direction inside the plane waveguide is described by the angle $\varphi$. For $\varphi=0^{\circ}$, the propagation is parallel to the KTP $z$-axis. In this case the TE modes are related to the $n_{y}$ component of the KTP refractive index tensor. For $\varphi=90^{\circ}$, propagation is parallel the KTP $y$-axis and the TE modes are related to $n_{z}$. In both configurations, the TM modes are related to $\mathrm{n}_{\mathrm{x}}$.

\section{Mode propagation in birefringent waveguides}

The $m$-line technique gives the effective indexes of the waveguide modes. Some theoretical considerations are necessary to deduce the waveguide geometry form the set of effective indexes. The index profile of ion-implanted waveguides can be approximated by a Fermi-type function. This type of index profile is best reconstructed using an inverse WKB method. In this method, a depth or turning point is attributed to each measured effective index [14], thus reconstructing the index profile (Fig. 2). In general, a few modes are sufficient to obtain the effective depth of the waveguide. The total index change is however difficult to calculate as the distinction between guided and leaky modes is not straightforward. This problem can be solved by supposing a step index profile (SIP), allowing to reconstruct the index profile with only two modes. In Fig. 2 the results of the two methods are compared. The index profiles are similar, although the SIP underestimates the waveguide depth, whereas the IWKB overestimates the surface refractive index. The effective indexes calculated from SIP (lines on the right of Fig. 2.) are in very good agreement to the experimental ones, and the TE 3 modes is clearly identified as leaky mode. In conclusion, the ion-implanted waveguides can be reasonably well approximated by a step index profile, facilitating the investigation of anisotropic waveguides.

Different approaches were applied to study the waveguiding properties of anisotropic planar waveguides, including the direct solution of the wave-propagation equation $[15,16]$ the finiteelement method [17], the zigzag ray model [18,19], approaches based on the separation of the waveguide modes into ordinary and extraordinary waves [20,21] and the transfer matrix model [2224]. It was found that for "off-axis" propagation the waveguide modes are of hybrid nature containing contributions of TE- and TM-type modes. Further on, for angles $\varphi$ greater than a critical angle, the dominantly TM-type modes are lossy modes with energy leakage into the substrate. The guided to leaky mode transition in uniaxial materials were identified near the ordinary or extraordinary cut-off for negative and positive birefringent materials respectively [25,26].

We developed a numerical simulation tool based on a transfer matrix approach [23] and using a step index profile. Our model allows the calculation of the effective indexes of the guided modes for any propagation direction in birefringent planar waveguides. The non linear Lenvenberg Marquardt method was used for fitting the waveguide parameters to the experimental results. The free Scilab environment was used for the calculations [27].

As already mentioned, pure TE and TM polarized modes only exist for propagation parallel to a dielectric axis of the material. For all other propagation directions the waveguide modes are hybrid modes containing TE and TM contributions. In most cases one contribution is, however, dominant. For simplicity, the labels TE' and TM' will be used for modes with dominant TE or TM 
contribution.

\section{Results and Discussion}

A typical $m$-line curve of ion implanted KTP waveguides is shown in Fig. 3 (dashed line). For convenience, the experimental incident beam angle was converted to the effective index using simple algebra. Two different phenomena are observed: three distinct $m$-lines related to the waveguide modes and a slow intensity decrease for decreasing effective indexes corresponding to light tunneling trough the low index barrier. The overlap of these two phenomena results in a slight shift of the minimum position of the $m$-lines and a decrease of their visibility. The pure $m$-line contribution are extracted by subtracting the envelop function which corresponds to the light tunneling (full line Fig. 3).

The $m$-lines obtained using prism and hemisphere couplers are compared in Fig. 4. Typical curves for TE and TM polarization are shown at $\varphi=0^{\circ}$. The minimal positions are in good agreement for the first two modes and excepting the first order mode, the contrast is equivalent. The hemisphere can thus be used without significant decrease of experimental precision.

The evolution of the $m$-lines curves with changing propagation direction is shown on Fig 5. As predicted by the theory, the TM' modes remains nearly unchanged, whereas the TE' modes are shifting towards higher effective indexes with increasing values of $\varphi$. The contrast of the $m$-lines does not change significantly with $\varphi$. Thus, the measurements with TE- and TM- polarized incident light beams allows the distinction of the hybrid TE' and TM' waveguide modes.

The effective indexes as a function of the propagation angle $\varphi$ are shown on Fig 6. All values were obtained using the hemisphere except for $\varphi=90^{\circ}$, where the prism was used. The experimental points were used to determine the waveguide structure by fitting to our theoretical model. A very good agreement between experiment and theory is observed up to the third order TE' or TM' modes. The TE' 4 mode could be resolved only for some angles. The good agreement of the higher order modes is surprising considering the large width of the respective $m$-lines (Fig. 5). The substrate indexes, index change, and thickness deduced from the theoretical fit are $: n_{\mathrm{x}}=1.7419, n_{\mathrm{y}}=1.7521$, $n_{\mathrm{z}}=1.8343, d=4.3 \mu \mathrm{m}$, and $\Delta n=0.025$, respectively. No significant anisotropy of the index change could be observed. Compared to the values obtained by fitting only the TE modes at $\varphi=90^{\circ}$ (Fig. 2), the index and index change are identical to the SIP model. The waveguide depth is, however, closer to the value of the IWKB model, which defines this parameter with better precision than the SIP model. In general, the precise determination of the waveguide parameters requires more than two fully guided modes in order to have a high ratio between the number of experimental points and the number of free parameters of the numerical fitting problem. Our results shows that a sufficient number of experimental points can also be obtained by varying the propagation direction in birefringent waveguides.

The good angular resolution of the hemisphere $m$-lines allows the investigation of the strong mode hybridization near the mode crossing in the $n_{\text {eff }}(\varphi)$ diagram (inset of Fig. 6). The theoretical model predicts that strong mode coupling is only possible for modes with opposite parity, e.g. TM'2 and TE'3. In this case the mode hybridization is total, resulting in the opening of a local effective index gap. In the case of equal parity modes (e.g. TM'1 and TE'3), the modes do not couple and no index gap can be observed. The experimental results seems to confirm this theoretical prediction. The 
precision of the presented results is actually not limited by the experimental method, but by the investigated waveguide. Narrower $m$-lines, and thus a better resolution, can be obtained by improving the light confinement inside the waveguide. This feature can be realized by increasing the index barrier height and width.

\section{Conclusions}

In conclusion we have shown that the hemisphere $m$-line spectroscopy is a useful tool for the characterization of birefringent planar waveguides. It allows the fast measurements of the effective indexes of the guided modes as a function of the propagation direction. The precision of the deduced waveguide parameters is enhanced and more advanced features such as the mode hybridization can be investigated. The presented results are only an example of possible applications of this technique. 


\section{References}

[1] P.K. Tien, R. Ulrich, R.J. Martin, Appl. Phys. Lett. 14 (1969) 291-294.

[2] R. Ulrich, J. Opt. Soc. Am. 60 (1970) 1337-1350.

[3] R. Reinisch, J. Fick, P. Coupier, J.L. Coutaz, G. Vitrant, Opt. Com. 120 (1995) 121-128.

[4] M. Nevière, E. Popov, R. Reinisch, G. Vitrant, Electromagnetic Resonances in Nonlinear Optics, Gordon and Breach Sci. Publ., Amsterdam, 2000.

[5] J. Fick, G. Vitrant, Opt. Lett. 20 (1995) 1462-1464.

[6] H. Rignault, F. Flory, S. Monneret, Appl. Opt. 34 (1995) 4358-4369.

[7] J. Fick, A. Martucci, M. Guglielmi, J. Schell, Fibre Int. Opt. 19 (2000) 43-56.

[8] A.V. Khomchenko, Opt. Com. 201 (2002) 363-372.

[9] G. Tartarini, P. Bassi, S. Chen, M.P. De Micheli, D.B. Ostrowsky, Opt. Comm. 101 (1993) 424431.

[10] P. Bindner, A. Boudrioua, J.C. Loulergue, P. Moretti, Nucl. Instr. Meth. Phys. Res. B 120 (1996) 88-92.

[11] M. Lindgren, J. Örtegren, P. Busson, A. Eriksson, D.S. Hermann, A. Hult, U.W. Gedde, P. Rudquist, S.T. Lagerwall, B. Stebler, Proc. SPIE 3475 (1998) 76-87.

[12] F. Flory, D. Edelema, E. Plletier, I. Hodgkinson, Appl. Opt. 32 (1993) 5649-5659.

[13] H. Jänchen, D. Edelema, N. Kaiser, F. Flory, Pure Appl. Opt. 5 (1996) 405-415.

[14] P. Mathey, P. Julien, Opt. Com. 122 (1996) 127-134.

[15] D. Marcuse, J. Quantum. Elec., QE-14 (1998) 736-741.

[16] D. Marcuse, I.P. Kaminov, J. Quantum. Elec., QE-15 (1979) 92-101.

[17] A.P. Zhao, S.,R. Cvetkovic, Opt. Lett. 20 (1995) 139-141.

[18] V. Ramaswamy, Appl. Opt. 13 (1974) 1363-1371.

[19] K. Hano, J. Opt. Soc. Am. A 4 (1987) 1887-1894.

[20] G. Tartarini, P. Bassi, P. Baldi, M.P. De Micheli, D.B. Ostrowsky, Appl. Opt. 34 (1995) 34413448.

[21] I. Avrutsky, J. Opt. Soc. Am. A 20 (2003) 548-556.

[22] A. Knoesen, T.K. Gaylord, M.G. Moharam, J. Lightw. Technol. 6 (1998) 1083-1104.

[23] T.A. Maldonado, T.K. Gaylord, J. Lightw. Technol. 14 (1996) 486-499.

[24] T.A. Maldonado, M. Ciumac, J. Lightw. Technol. 15 (1997) 1747-1755.

[25] L. Torner, J. Recolons, J.P. Torres, J. Lightw. Technol. 11 (1993) 1592-1600.

[26] M. Lu, M.M. Fejer, J. Opt. Soc. Am. A 10 (1993) 246-261.

[27] http://www.scilab.org 


\section{Figure Captions}

Figure 1: Experimental hemisphere $m$-line set-up.

Figure 2: Reconstruction of the waveguide geometry using the measured TE modes for $\varphi=90^{\circ}$. The full line corresponds to the step index calculation and the dashed line and squares to the IWKB method. At the right of the figure are shown the effective indexes values calculated from the step index profile.

Figure 3: TE $m$-line curve at $\varphi=0^{\circ}$ : dashed line (left y-axis) original curve, continuous line (right $\mathrm{y}$-axis) curve after envelope subtraction.

Figure 4: Comparison between $m$-line curves obtained with coupling prism (continuous) and the hemisphere curves (dashed) for TE and TM polarization at $\varphi=0^{\circ}$.

Figure 5: Evolution of $m$-lines for $\varphi$ in between 0 and $40^{\circ}$ for TE- (continuous) and TM-(dashed) polarized incident beam.

Figure 6: Evolution of the effective indexes as a function of propagation angle $\varphi$. The circles corresponds to the experimental points (full : TM', empty : TE'), the lines to the theoretical model. The different modes are indexed at $\varphi=90^{\circ}$. The inset shows a zoom of the $\varphi=10^{\circ}$ to $30^{\circ}$ region. 


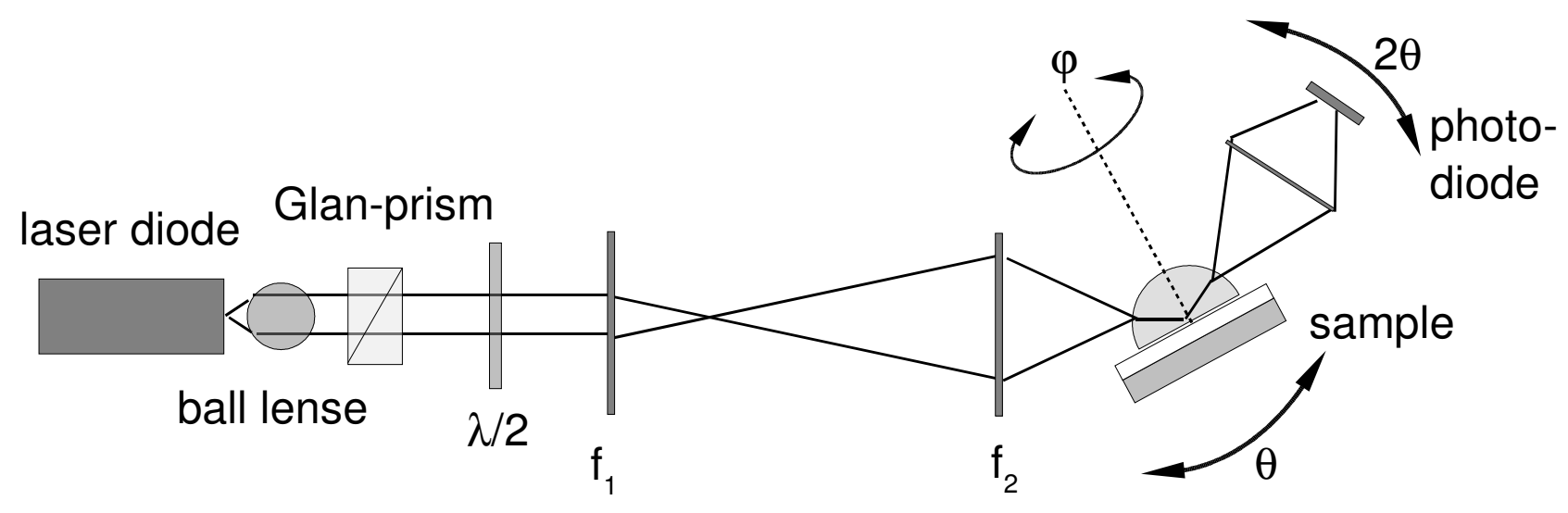

Figure 1

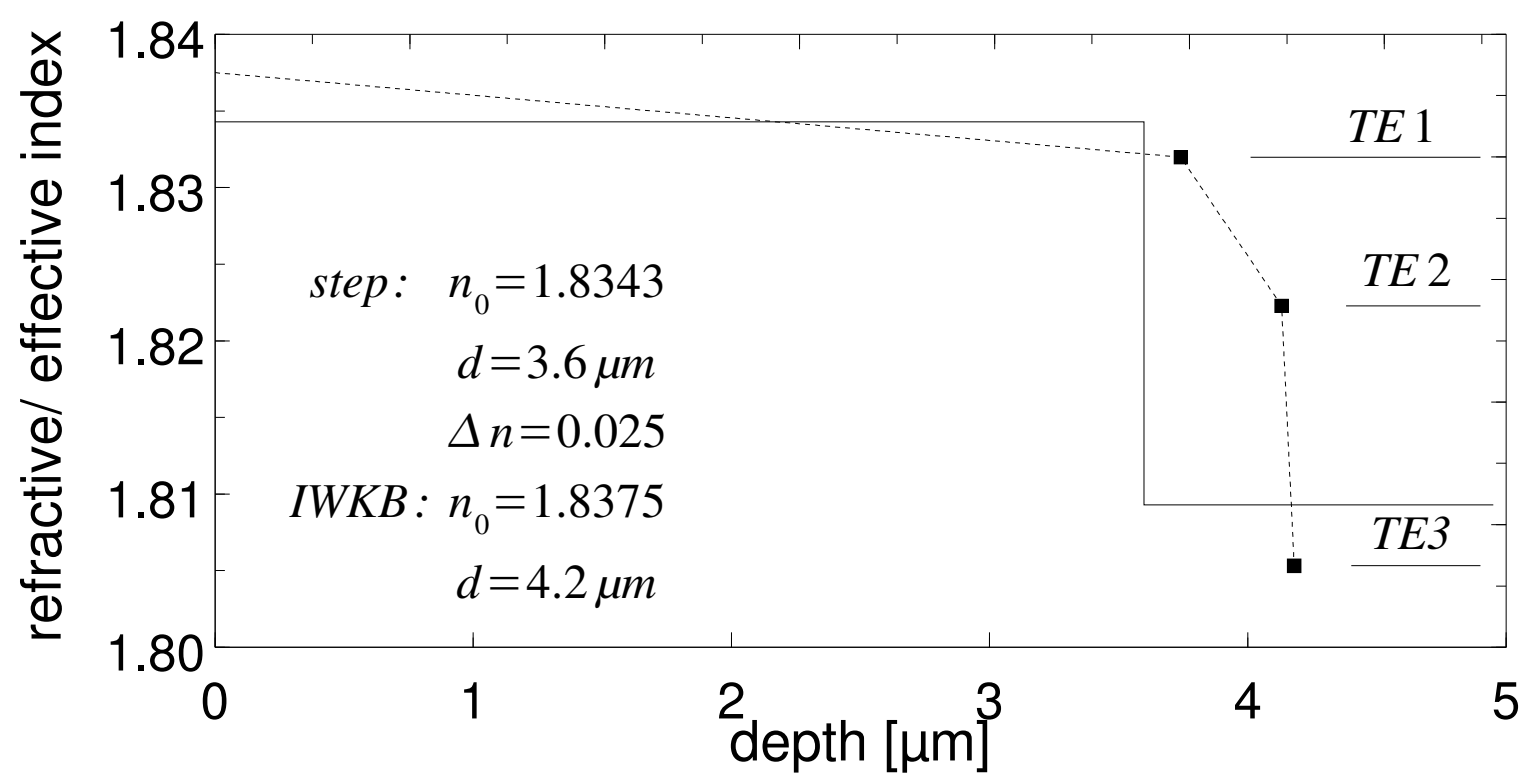

Figure 2 


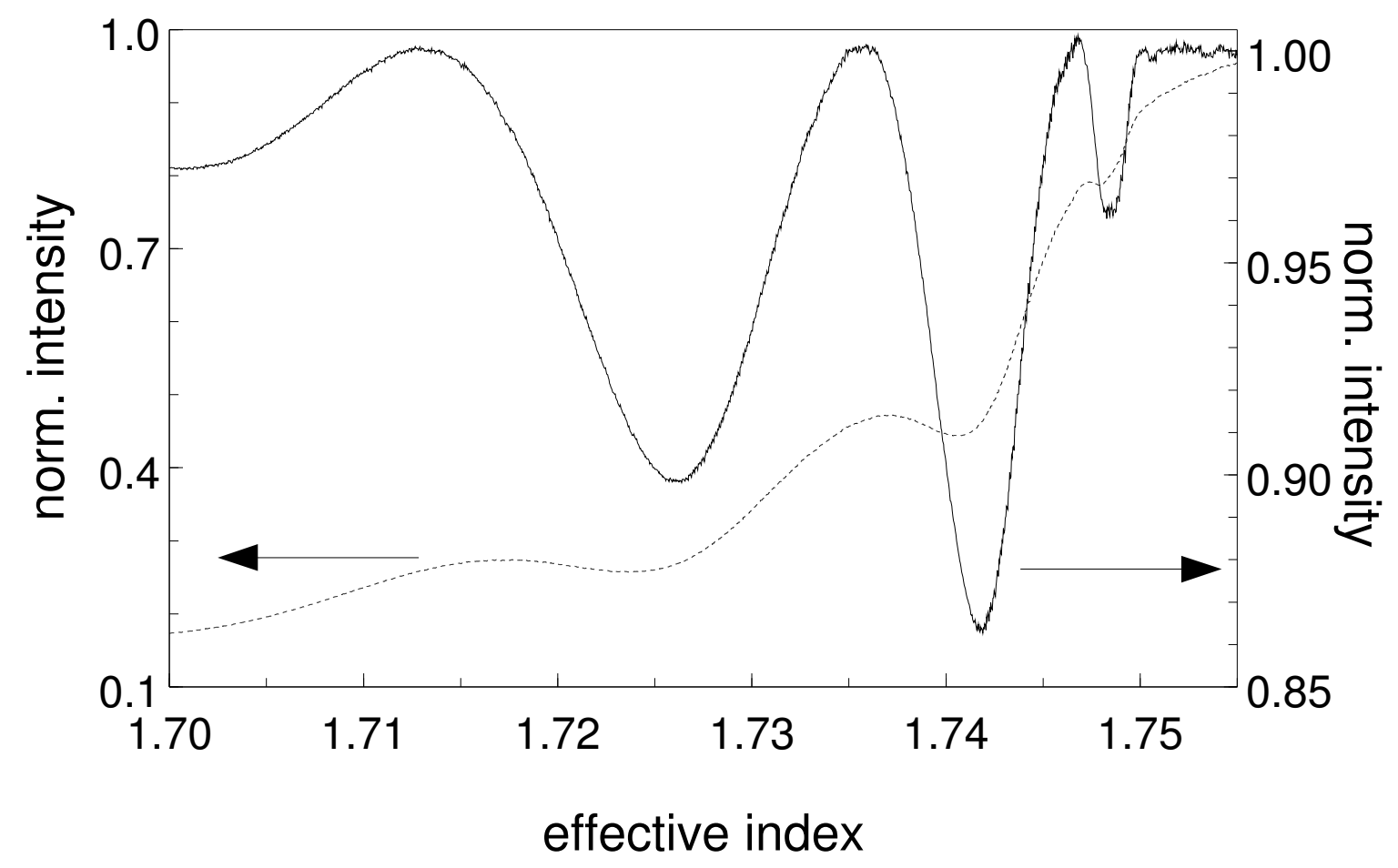

Figure 3

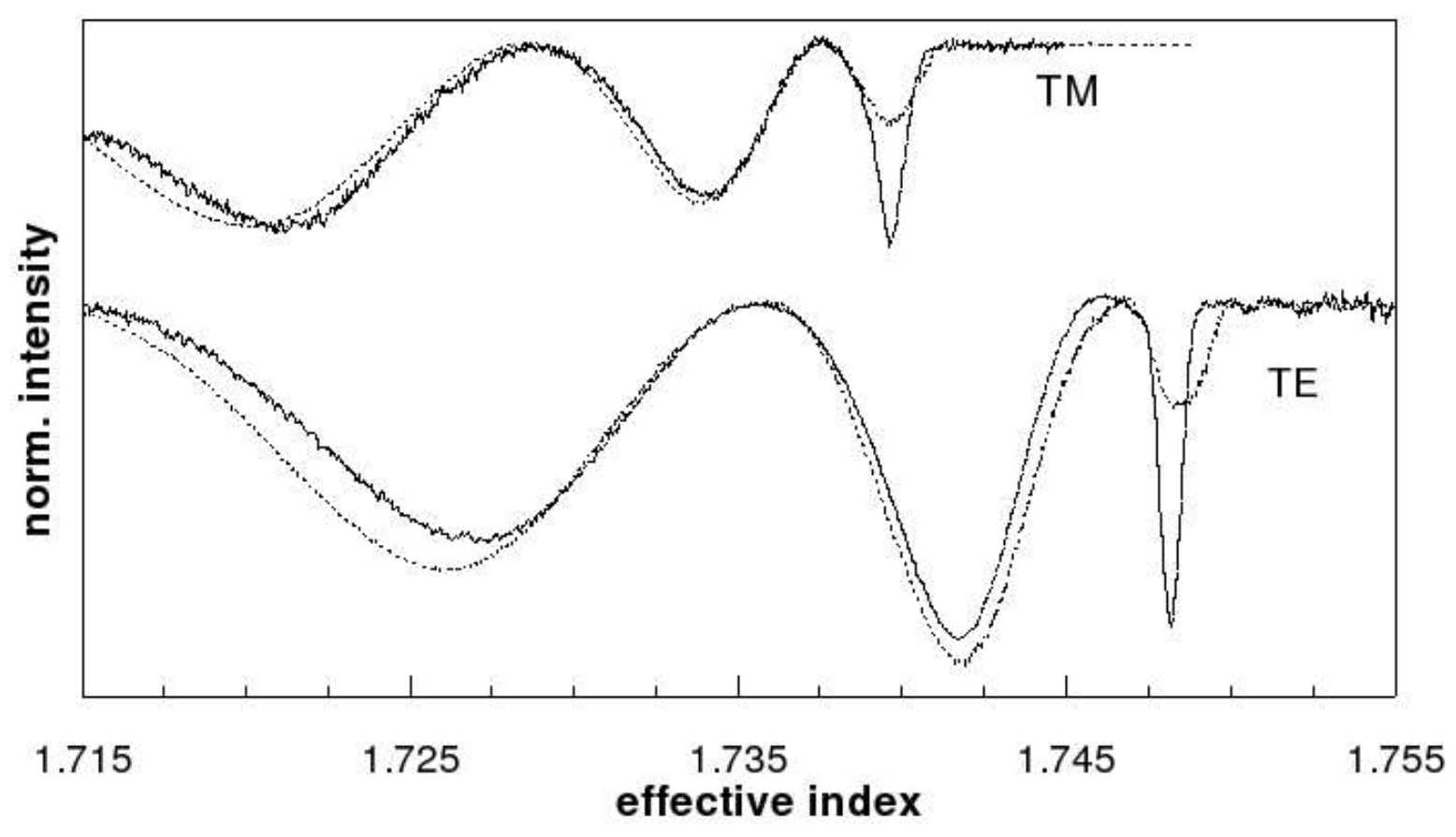

Figure 4 


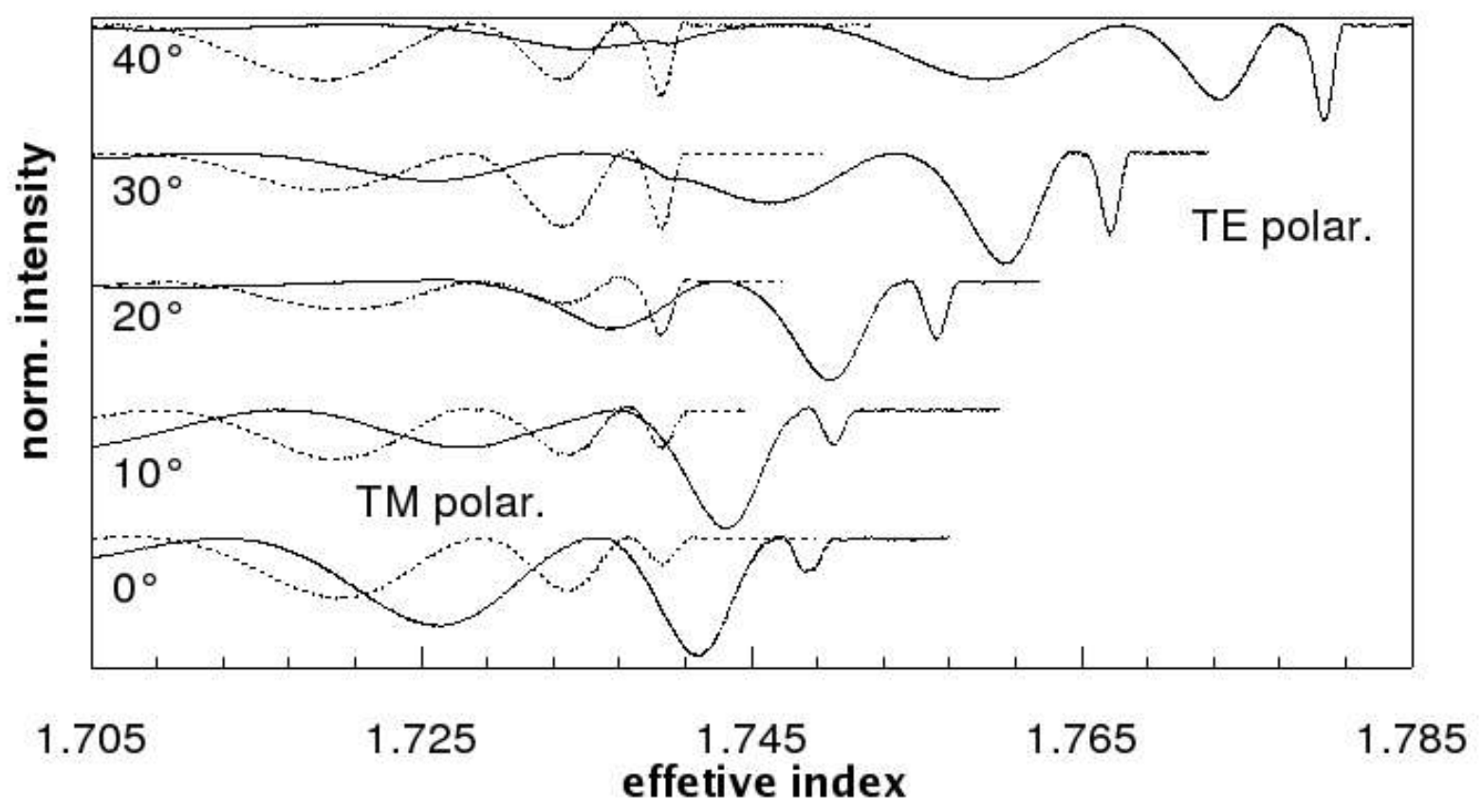

Figure 5 


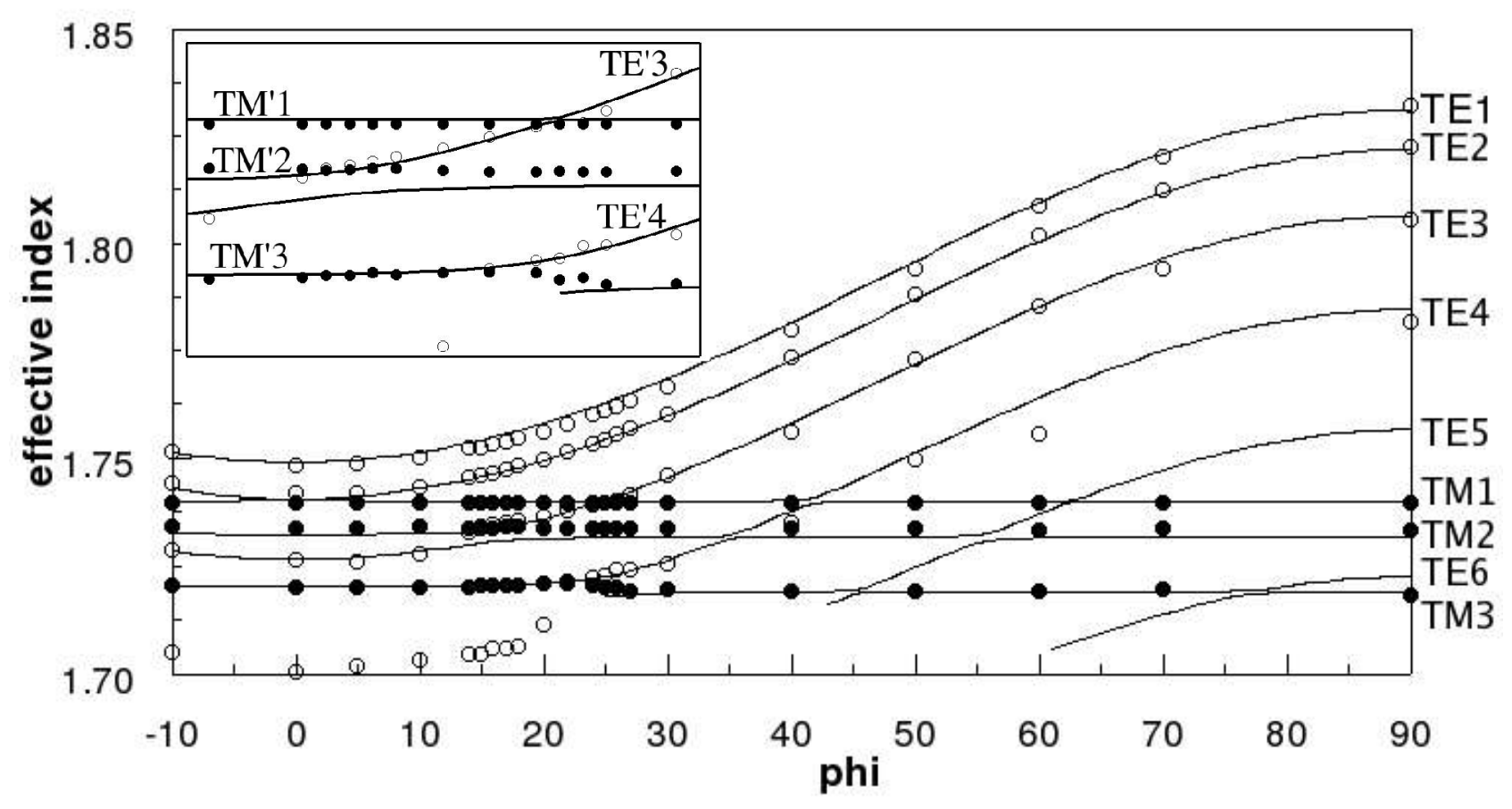

Figure 6 